\title{
A new technique to reproduced high-dynamic-range images for low-dynamic-range display
}

\begin{abstract}
Tone mapping is a process for reproduction of High-Dynamic-Range images (HDR) for LowDynamic-Range (LDR) output devices. In this report, author presents a new local tonemapping operator, derived from the Contrast Limited Adaptive histogram Equalization (CLAHE) technique for displaying high dynamic range image. The CLAHE is a method which was originally developed for medical imaging. This method has effectively expanded the full dynamic range of display and it is fully automatic. Due to different luminance intervals could result in overlapped reaction on the limited response in limited response range of visual system, scene region splitting and merging were used to segment the scaled luminance and perform the image segmentation to segment image into smaller part. After the region splitting and merging, there will be some noise or variation of intensity that may result in holes or over segmentation. As the result, the morphological operation, opening and closing were used to perform the mask to applied different clip limit of the CLAHE operation.
\end{abstract}

Keyword: CLAHE; High-dynamic-range; Image; Luminance; Tonemapping 